\title{
The Effect of Roll and Pitch Motion on Ship Magnetic Signature
}

\author{
Marius Birsan $^{1 *}$ and Reinier Tan ${ }^{2}$ \\ ${ }^{1}$ Defence R\&D Canada - Atlantic, Dartmouth, NS, Canada \\ ${ }^{2}$ TNO, The Hague, Netherlands
}

(Received 4 October 2016, Received in final form 12 December 2016, Accepted 13 December 2016)

\begin{abstract}
The roll- and pitch-induced eddy currents create a magnetic field that contributes to the total magnetic signature of naval vessels. The magnetic signature is of concern, as it exposes the ship to the threat of modern influence mines. It is estimated that the eddy current is the second most important source contributing to a ship's underwater magnetic field following the ferromagnetic effect. In the present paper, the finite element (FE) method is used to predict the eddy current signature of a real ship. The FE model is validated using the measurements of the Canadian research vessel CFAV QUEST at the Earth's Field Simulator (EFS) in Schirnau, Germany. Modeling and validation of the eddy current magnetic signature for a real ship represents a novelty in the field. It is shown that the characteristics of this signature depend on frequency. Based on these results, a ship's degaussing system could be improved to cancel both the ferromagnetic and the eddy current contribution to the magnetic signature simultaneously, reducing the susceptibility to sea mines.
\end{abstract}

Keywords : eddy currents, ship signature, finite element method

\section{Introduction}

Eddy currents are induced in electrically conducting materials onboard naval vessels as they roll and/or pitch within the Earth's magnetic field. These eddy currents create a magnetic field in reaction.

Degaussing systems onboard naval vessels have been used for more than 70 years to reduce the static magnetic signature and thus reduce the threat of influence mines. However, due to the development in sensor technology, the emission of the alternating electric and magnetic fields have become a concern in electromagnetic silencing of ships [1]. For example, in high sea states, the ship magnetic signature can vary considerably from those in calm seas. This has implications for magnetic influence mine actuation due to the additional magnetic field produced by the eddy currents. Ships constructed from nonmagnetic conducting metals, such as aluminum or stainless steels, will also generate eddy current magnetic signatures, so they may also be detected by the magnetic sensor inside the influence mine. Thus, modeling and measurements of alternating magnetic signatures should

CThe Korean Magnetics Society. All rights reserved.

*Corresponding author: Tel: +1-902-426-3100 x 375

Fax: +1-902-426-9654, e-mail: marius.birsan@drdc-rddc.gc.ca be a research priority in maritime engineering.

A previous study [2] used a simple two-dimensional model to predict the eddy currents magnetic signature with no validation. According to this model, in certain conditions, the magnetic signature produced by eddy currents can be significant in comparison to the ferromagnetic signature. This makes eddy currents an important magnetic source contributing to a ship's underwater field.

Aside from the above study that used an infinite cylindrical conducting shell to model the eddy current signature of naval vessels, no other investigations have been carried out. Such investigations are necessary because a successful magnetic silencing of the ship strongly depends on how to minimize the individual field components produced by different magnetic sources.

A naval vessel is typically constructed of ferrous steel plates and has complicated internal structures, such as bulkheads, engines, and various steel-manufactured equipment, in addition to the hull. For this reason, it is very difficult to predict the field disturbance due to the eddy currents using some analytical models. The only feasible way to predict this signature is with numerical methods, such as the finite element method. However, a numerical model is useful only when it is validated by measurements. Therefore, an accurate measurement method on the magnetic fields produced by induced eddy currents is 
required.

For a reliable measurement of the eddy currents magnetic signature, a suitable measurement facility must be available. Such a facility is the German Earth's Field Simulator (EFS) in Schirnau, Germany. The facility is run by the German naval institute WTD-71. The EFS is a fixed facility constructed with a set of coils that surround the vessel and used to induce a magnetization in each of the three orthogonal directions. Beneath the ship there are two 2-D arrays of magnetic field sensors at a depth of 9 $\mathrm{m}$ and $13 \mathrm{~m}$. After the ship is moored within the EFS, the current in each coil is controlled to create the desired simulation of the ship attitude (position, heading, roll, and pitch).

Without such a facility, the evaluation of the motionproduced eddy currents effect for a certain ship relies on models. It is also possible that the dimensions of the ship exceed the EFS dimensions, which means that the ship signature produced by eddy currents needs to be estimated from models.

The Earth's Field Simulator in combination with the finite element method was previously used to investigate the ship signature produced by the permanent magnetization [3]. In this study, a physical scale model has been employed to validate the finite element model.

During the RIMPASSE trial [4] in 2011, the roll and pitch movements were simulated when the Canadian Forces Auxiliary Vessel (CFAV) QUEST was at the EFS, and their effects measured by sensors under the ship. These measurements will be compared with the results obtained from the finite element model of the vessel. Having a validated model is important because it can be applied to simulate the ship signature in various scenarios.

The goal of this paper is to present a model validation for the eddy current magnetic signature produced by a real ship. The present measurements and modeling represent a novelty in the field.

\section{Earth's Magnetic Field Simulator}

The magnetic Earth Field Simulator is designed to simulate any magnetic field that naval vessels may face worldwide and any additional magnetic conditions, including the eddy current simulations. The facility is used primarily to measure the magnetic signature of German submarines and mine hunters and, subsequently, to obtain proper settings for the degaussing system. During the RIMPASSE trial CFAV QUEST was in fact the first ship with a ferromagnetic hull to be measured at the facility.

The Earth Field Simulator consists of 14 longitudinal coils, 3 athwartship coils, and 3 vertical coils as shown in

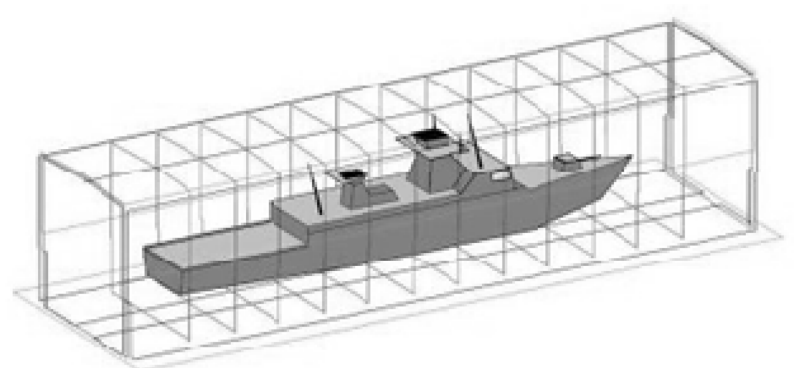

Fig. 1. Schematic diagram of the Earth's Field Simulator.

Fig. 1. The longitudinal, or X, coils orientation corresponds to the magnetic south-north direction; the athwartship, or $\mathrm{Y}$, coils to the west-east direction and the vertical coils to the $\mathrm{Z}$ direction (downwards). This coil configuration provides the ability to simulate the magnetic field at any place on the earth, and at any heading and ship movement. It also provides the ability to zero the background field and measure the vessel's permanent magnetic signature directly.

Tri-axial magnetic probes are aligned in a grid of 36 by 94 meters below the vessel at two levels, 9 and $13 \mathrm{~m}$ from waterline, to measure the response of the vessel under different magnetic conditions. On each of the two levels, there are 29 sensors rows along the X-axis with 13 sensors on each row. The sensors orientation corresponds to the orientation of the coils.

The water depth in the simulator is about $8 \mathrm{~m}$. The simulator has a length of $100 \mathrm{~m}$, width of $21 \mathrm{~m}$ at the entrance, and a height of $25 \mathrm{~m}$ from the water surface.

The components of the Earth's magnetic field at the measurement location are $17.7 \mu \mathrm{T}$ in the north-south direction $\left(\mathrm{B}_{\mathrm{x}}\right)$, and $46.5 \mu \mathrm{T}$ in the vertical direction $\left(\mathrm{B}_{\mathrm{z}}\right)$.

The displacement of CFAV QUEST is 2200 tons and the ship dimensions are $76 \mathrm{~m}$ length, $12.6 \mathrm{~m}$ beam, and $4.8 \mathrm{~m}$ draft. The ship was able to enter the magnetic field simulator after the removal of the upper part of the mast.

In the ship coordinate system, the $\mathrm{X}$ axis corresponds to the longitudinal direction oriented forward, $\mathrm{Y}$ axis to the athwartship direction oriented to starboard, and $\mathrm{Z}$ axis is perpendicular to both $\mathrm{X}$ and $\mathrm{Y}$, pointing downwards.

At the measurement station, the ship was oriented with its axes parallel to the axes of the magnetic field simulator. The ship's locations from the magnetic sensors were measured very accurately with a Laser Range Finder, which has a distance measurement error of less than $1 \mathrm{~cm}$.

For each magnetic condition under which CFAV QUEST was measured, the same condition had to be measured without the ship in the simulator. These data were subtracted from the measurements made with CFAV QUEST 
in the simulator to yield only the ship's signature. The sample rate for static field measurements was $20 \mathrm{~Hz}$ and the sampling from all sensors was synchronized.

During the RIMPASSE trial, the following measurements related to the eddy currents signature were performed on CFAV QUEST at the EFS:

Variable sine wave magnetic fields were applied along each axis with amplitude of $20 \mu \mathrm{T}$ peak-to-peak and frequencies of $1 / 5 \mathrm{~Hz}, 1 / 8 \mathrm{~Hz}$ and $1 / 16 \mathrm{~Hz}$.

Simulations of the roll motion with the ship heading West in the Earth's magnetic field of $(0,17.7,46.5) \mu \mathrm{T}$, roll angle \pm 15 , and frequencies of $1 / 8 \mathrm{~Hz}$ and $1 / 16 \mathrm{~Hz}$.

Simulations of the pitch motion with the ship heading North in the Earth's magnetic field of $(17.7,0,46.5) \mu \mathrm{T}$, pitch angle $\pm 15^{\circ}$, and the frequencies of $1 / 8 \mathrm{~Hz}$ and $1 / 16$ Hz.

\section{Modeling the Eddy Current Magnetic Signature}

The finite element (FE) method is commonly used to compute the effect of the Earth magnetic field on large ships [5], including their roll and pitch movements. Such calculations, however, need to be validated by measurements. Once the model is validated, the FE model could predict the vessel signature for a variety of scenarios including different magnetic locations, headings, roll and pitch angles and frequencies.

The finite element model of CFAV Quest (Fig. 2) was created in FLUX-3D, a commercial software product. The model was primarily used to calculate the ship's magnetic induced signature, but it could be easily modified to calculate the eddy currents inside the metal plates and the related magnetic fields. As the model was initially created for structural analysis, it accurately represents the structures of the ship. Moreover, the model includes the large metallic masses representing the engines (Diesel electric and gas turbine).

One can define the magnetic anomaly created by the eddy currents with respect to the original field as the

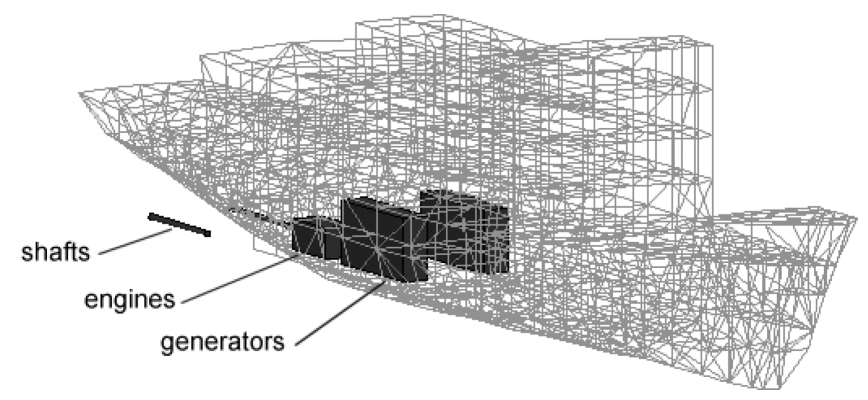

Fig. 2. Finite element model of CFAV QUEST. difference between the total resultant field and the original source field. A reduced magnetic scalar potential $\left(\varphi_{r}\right)$ formulation related to the source fields, $\mathbf{H}_{j}$, has been developed in FLUX-3D [6]. The advantage of this formulation is a simple computation of the magnetic anomaly $\left(\mathbf{B}_{a n}\right)$ outside the ferromagnetic material:

$$
\mathbf{B}_{a n}=\mu_{0}\left(\mathbf{H}-\mathbf{H}_{j}\right)=-\mu_{0} \nabla \varphi_{r}
$$

where $\mu_{0}$ is the permeability of free space, and $\mathbf{H}$ is the total magnetic field.

In order to create relative motion between the ship and the Earth's magnetic field, the whole FE geometrical problem is fixed and the sources are varied with time.

The first case assumes that the external sine wave timevarying magnetic field is applied along one axis. When the measurements are carried out, the electric current signal that excites the source coil can be used as the reference for the phase calculation. In this case, the two temporal components of the eddy current signature, the real and the quadrature components, can be separated. The quadrature component is due to the lag in the magnetization response to the applied magnetic field.

The second case defines a single FE simulation that consists of one external source and one type of movement. The FE method described here considers only the roll of the ship in the vertical magnetic field, $H_{V}$ [5].

The angular position of the ship during the roll movement depends on time according to the relation:

$$
\alpha=\alpha_{0} \cos (\omega t)
$$

where $\alpha_{0}$ is the maximum roll angle, $t$ is time, and $\omega$ is the angular roll frequency. The vertical field $H_{V}$ is projected on the ship's coordinate system and then the Taylor's expansion is used:

$$
\begin{aligned}
H_{Y} & =H_{V} \sin \left(\alpha_{0} \cos (\omega t)\right) \cong H_{V} \alpha_{0} \cos (\omega t) \\
H_{Z} & =H_{V} \cos \left(\alpha_{0} \cos (\omega t)\right) \cong H_{V}-H_{V} \frac{\alpha_{0}^{2}}{2} \cos ^{2}(\omega t)= \\
& =H_{V}-H_{V} \frac{\alpha_{0}^{2}}{4}-H_{V} \frac{\alpha_{0}^{2}}{4} \cos (2 \omega t)
\end{aligned}
$$

This type of movement-induced eddy current signature simulation involves two frequencies, $\omega$ and $2 \omega$, so that the phase reference signal cannot be defined.

Situations where other components of the external field are present and/or the ship oscillates around different axes have to be divided into separate simulations. As the external sources and the ship movements are independent, the final signature result is equal to the summation of partial results from each simulation.

The meshing of the ship has to be small enough (a few 
centimetres, in this case) to enable the correct calculation of the eddy currents on each surface. The computation time for a simulation is about two hours on a 12-core, 3.2 $\mathrm{GHz}, 24$ GB RAM computer, thus the prediction of the signature cannot be done in real time. This is not necessary, however, as for inclusion of the eddy current signature in the degaussing system the calculations only need to be done once for each relevant frequency.

\section{Model Validation}

The model was validated in two steps: (i) using the uniaxial fields in $\mathrm{X}, \mathrm{Y}$, and $\mathrm{Z}$ directions, the electric conductivity and the permeability of the model were adjusted so that the measured data could be reproduced with reasonable accuracy, and (ii) the model was verified against the measurements obtained from a combination of sources, as in roll and pitch simulations.

Note that FLUX-3D does not incorporate an optimization module, so that the model parameters were determined by trial and error. The model was considered to fit the data for an overall relative permeability of 200 and an electrical conductivity specific to steel of $5.0 \mathrm{MS} / \mathrm{m}$. The properties of the engines were modeled separately with a relative permeability of 50 and conductivity of $5.0 \mathrm{MS} / \mathrm{m}$. The comparison between the calculated and measured data at $13 \mathrm{~m}$ depth along the keel line is shown in Figures 3 to 6 . The zero position on the graphs corresponds to the ship mid-point, so that the stern is at $-38 \mathrm{~m}$.

Figures 3 and 4 present the in-phase (real) and out-ofphase (imaginary) root mean square (rms) values of the eddy current induced magnetic field in the $\mathrm{X}$ direction $\left(\mathrm{B}_{\mathrm{X}}\right)$ when $\mathrm{X}$-coils were activated. The behavior of the in-

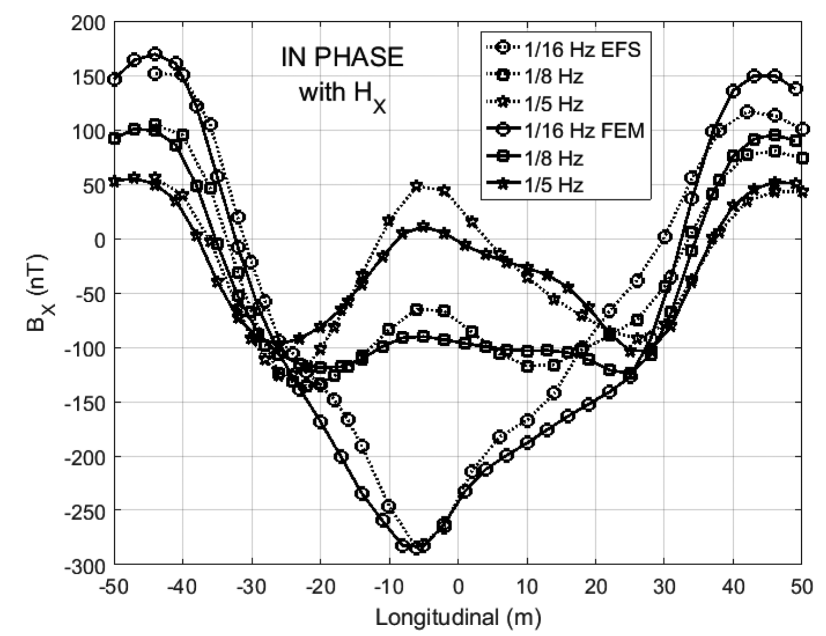

Fig. 3. The in-phase component of the longitudinal eddy current induced magnetic field at $13 \mathrm{~m}$ depth under the keel when sine wave $H_{x}$ was applied.

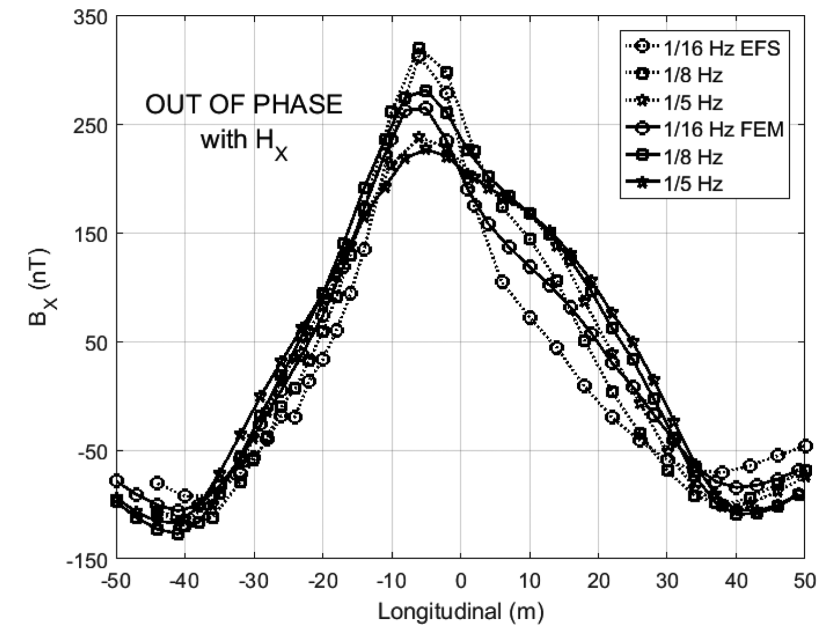

Fig. 4. The out-of-phase component of the longitudinal eddy current induced magnetic field at $13 \mathrm{~m}$ depth under the keel when sine wave $H_{x}$ was applied.

phase component changes with frequency. At the lowest frequency $(1 / 16 \mathrm{~Hz})$, the equivalent magnetic source corresponding to this component is oriented in the positive longitudinal direction. At higher frequencies, there is a tendency for this equivalent magnetic source to split into two sources of the same polarization. The equivalent magnetic source producing the out-of-phase component is oriented in the negative longitudinal direction.

A different behavior is exhibited in the in-phase component of the eddy current induced longitudinal magnetic field, $\mathrm{B}_{\mathrm{X}}$, when the ship is subject to a vertical field, as shown in Fig. 5. With increasing frequency, the polarization of the equivalent magnetic source corresponding to the induced eddy currents changes sign from plus to minus. The plots show clearly the effect of the induced

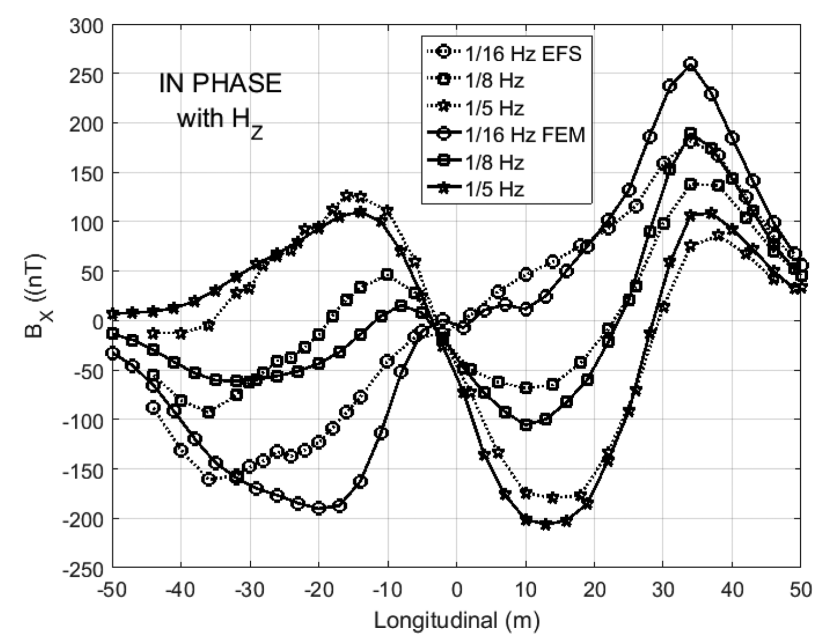

Fig. 5. The in-phase component of the longitudinal eddy current induced magnetic field at $13 \mathrm{~m}$ depth under the keel when sine wave $H_{z}$ was applied. 


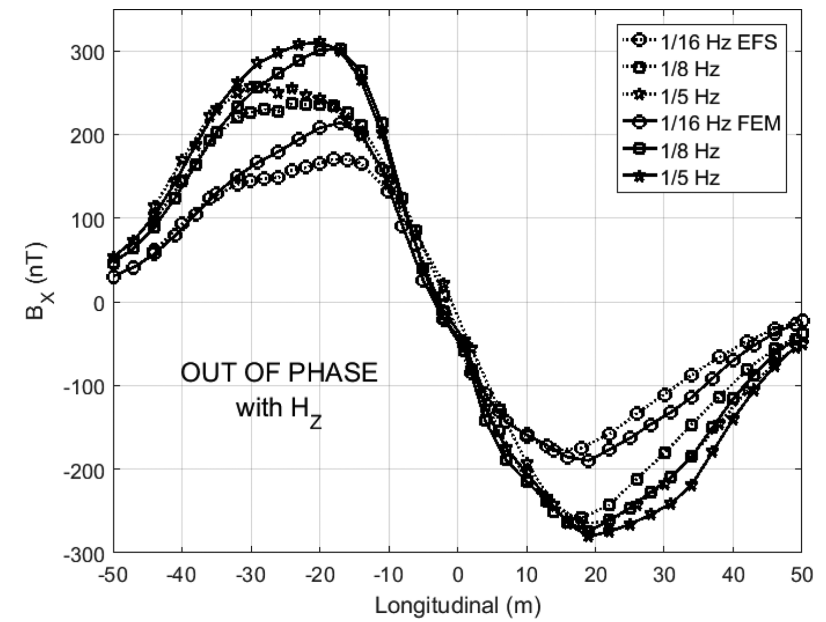

Fig. 6. The out-of-phase component of the longitudinal eddy current induced magnetic field at $13 \mathrm{~m}$ depth under the keel when sine wave $\mathrm{Hz}$ was applied.

eddy currents, which, with increasing magnetic field frequency, tend to exclude the magnetic field from the conducting material.

As before, the equivalent magnetic source producing the out-of-phase component preserves its polarization.

To verify the model, the magnetic signatures are computed with the ship assumed to roll with an angle of $\pm 15^{\circ}$ while it is heading west. The time-varying magnetic fields inside the simulator, in $\mathrm{A} / \mathrm{m}$ amplitude values, created in the $\mathrm{Y}$ and $\mathrm{Z}$ directions are not sine waves, but are given by:

$$
\begin{aligned}
& H_{Y}=9.62 \sin (\omega t)+0.24 \sin (2 \omega t) \\
& H_{Z}=3.68 \sin (\omega t)+0.63 \sin (2 \omega t)
\end{aligned}
$$

The measurement of the anomaly was performed by separating the time-varying component from the total signature at $13 \mathrm{~m}$ depth, and calculating its amplitude. The phase of the signal was not considered, so that the amplitude represents the sum of real and imaginary components.

The results of the calculation and the measurement data, which represents the amplitude values of the timevarying signal at the sensors in the Z-direction for 1/16 $\mathrm{Hz}$ frequency, are presented in Fig. 7 and 8. In the plots, the ship bow is at $(x=0, y=38) \mathrm{m}$. The asymmetry of the signature is due to the overlap of the effects created by the simultaneous application of the $H_{Y}$ and $H_{Z}$ components of the field (in eq. 4).

It was observed that the magnetic signatures generated by eddy currents measured at the EFS for the two frequencies, $1 / 8 \mathrm{~Hz}$ and $1 / 16 \mathrm{~Hz}$, of the ship rolling are similar, both in shape and amplitude. This is due to the fact that, even at a higher frequency $(1 / 8 \mathrm{~Hz})$ the in-phase

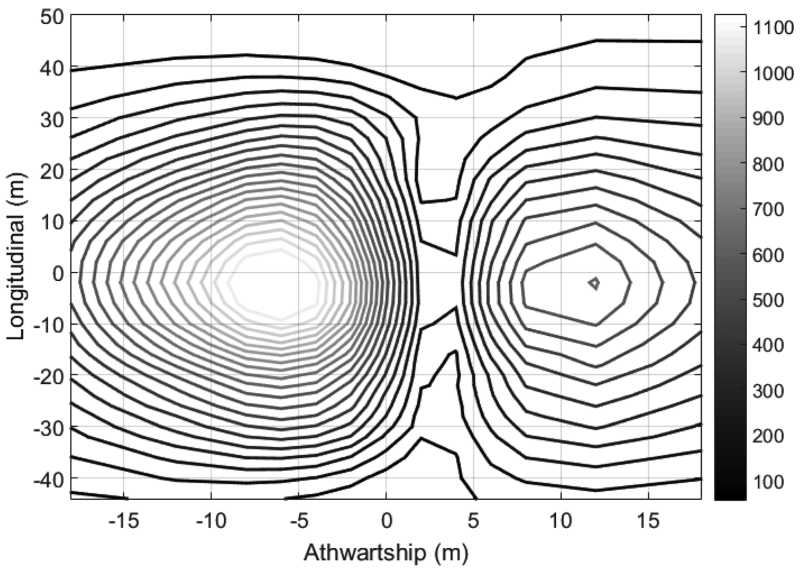

Fig. 7. Measured $B_{z}$ eddy current signature produced by roll motion $\left( \pm 15^{\circ}\right.$ at $\left.1 / 16 \mathrm{~Hz}\right)$.

component decreases as compared with its lower frequency $(1 / 16 \mathrm{~Hz})$ value, the out-of-phase component increases. Similar considerations apply to the pitch movements.

As shown in Fig. 7, at the low frequencies where the measurements were taken, the magnetic signatures generated by the roll-induced eddy currents have the same characteristics as the undegaussed vertical coupled with athwartship ferromagnetic signatures. Correspondingly, the pitch-induced eddy currents have equivalent magnetic sources primarily oriented in the vertical and longitudinal directions. Consequently, these magnetic anomalies can, in principle, be cancelled with degaussing coils.

The measurements at the EFS were important in understanding the mechanism of eddy currents induced magnetic signature in a real ship in order to determine the appropriate measures to compensate it. From the practical point of view, the frequencies of the magnetic fields used to simulate the roll and pitch movements of CFAV

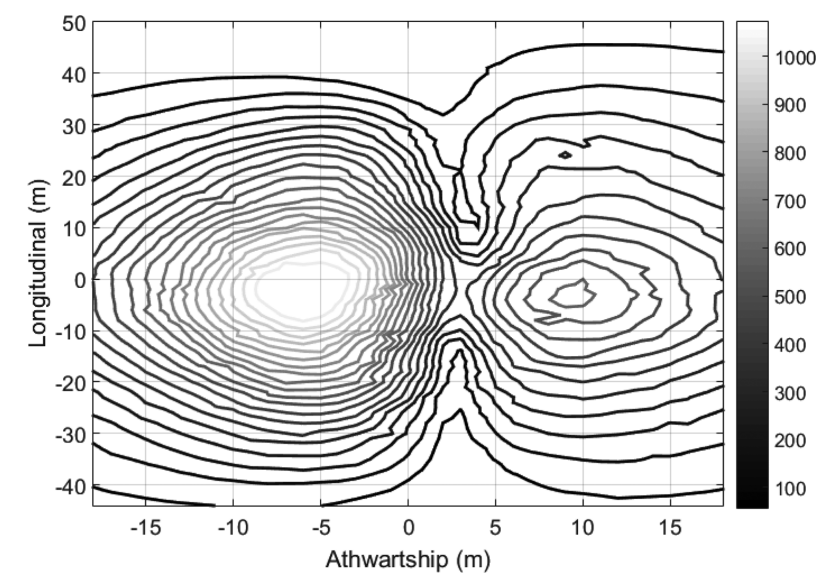

Fig. 8. Calculated $B_{z}$ eddy current signature produced by roll motion $\left( \pm 15^{\circ}\right.$ at $\left.1 / 16 \mathrm{~Hz}\right)$. 
QUEST at the EFS were close to the real values. Simulation of the ship movement based on the Computational Fluid Dynamics method has shown that the natural roll frequency of CFAV QUEST is approximately $1 / 8 \mathrm{~Hz}$. In sea state 5 at a speed of 15 knots, the same modeling shows that the roll angles do not exceed $\pm 5^{\circ}$. However, at slower speed at headings perpendicular to the direction of the waves, the ship rolls more. At 5 knots (in sea state 5) roll angles go up to $12^{\circ}$. In this case, the finite element model predicts a $B_{z}$ roll-induced signature similar to the one shown in Fig. 8.

\section{Conclusions}

The fields produced by eddy currents were measured for the first time in the Earth's Field Simulator using a real ship. These fields may have a significant contribution to the ship magnetic signature, especially if the ship is degaussed.

Because the sources that generate the eddy currents and the ferromagnetic signatures are independent, their effect is cumulative. These sources can be treated in a similar way with the result that the eddy current signature could be cancelled with the existing degaussing coils.
Using the finite element model of the ship, the measured signatures produced by eddy currents can be reproduced with reasonable accuracy. Once the model is validated, it can predict the roll- and pitch-induced magnetic signatures for any realistic scenario of ship movements at any depth of water.

\section{References}

[1] Z. Wu, X. Zhu, and Bin Li, Sensors \& Transducers 186, $161(2015)$.

[2] J. Holmes, Reduction of a Ship's Magnetic Field Signatures, Synthesis Lectures on Computational Electromagnetics, Morgan \& Claypool (2008).

[3] H.-J. Chung, C.-S. Yang, and W.-J. Jung, J. Magn. 20, 62 (2015).

[4] NATO STO Technical Report, Signature Management System for Underwater Signatures of Surface Ships, TRSET-166 (2015).

[5] X. Brunotte, G. Meunier, and J.-P. Bongiraud, Fifth Biennial IEEE Conference on Electromagnetic Field Computation, 32 (1992).

[6] Y. Le Floch, C. Guerin, X. Brunotte, and G. Meunier, IEEE Trans. Magn. 38, 553 (2002). 